\title{
CAPUTO FRACTIONAL INTEGRO-DIFFERENTIAL EQUATION WITH NONLOCAL CONDITIONS IN BANACH SPACE
}

\author{
Mohammed S. Abdo ${ }^{1}$, Abdulkafi M. Saeed ${ }^{2}$, Satish K. Panchal ${ }^{3}$ \\ ${ }^{1,3}$ Dr. Babasaheb Ambedkar Marathwada University \\ Aurangabad 431004 (M.S.), INDIA \\ ${ }^{2}$ Dep. of Mathematics \\ College of Science \\ Qassim University \\ Buraydah 51452, SAUDI ARABIA
}

\begin{abstract}
The aim of the present paper is to prove the existence of solutions of the initial value problem for a nonlinear integro-differential equation of fractional order $\alpha \in(0,1)$ with nonlocal conditions in Banach spaces. The fractional differential operator is taken in the Caputo sense. The presented analysis relies on the Krasnoselskii's fixed point theorem. At the end, an illustrative example will be introduced to justify our result.
\end{abstract}

AMS Subject Classification: 34A08, 26A33, 46E15, 47H10

Key Words: fractional integro-differential equation, fractional derivatives and integrals, Banach space, fixed point theorem

\section{Introduction}

Fractional differential equations are joined with intensive applications such as continuum phenomena mechanics, electrochemistry, biophysics, biotechnology engineering etc. For a lot of details see studies of Kilbas et al. [13], Miller and Ross [14], Samko et al. [16], and plenty of different references. The existence and uniqueness of solutions to fractional differential equations have attracted

Received: December 26, 2018

(c) 2019 Academic Publications

${ }^{\S}$ Correspondence author 
the attention of many scientists and researchers, as an example, (see $[2,3,4,10$, $11,12])$. In recent analysis work, several researchers of mathematics community studied fractional integro-differential equations because of their applications in different fields of science and engineering, for a lot of data see associate, $[1,5,6,7,8,9,15]$. Nonlocal conditions come up once values of the function on the boundary are connected to values within the domain. It is found to be a lot of plausible than the standard initial conditions for the formulation of some physical phenomena in certain problems of thermodynamics, elasticity and wave propagation. In passing, we have a tendency to noticed that nonlocal condition $u(0)=\sum_{k=1}^{m} c_{k} u\left(\tau_{k}\right)$ can be applied in physical problems yields better effect than the initial conditions $u(0)=u_{0}$.

The purpose of this paper is to prove the existence of solutions of the following nonlinear fractional integro-differential equations in Banach space

$$
{ }^{c} D_{0^{+}}^{\alpha} u(t)=a(t) u(t)+h(t)+\int_{0}^{t} K(t, \sigma) f(u(\sigma)) d \sigma, \quad t \in[0, b],
$$

with nonlocal conditions

$$
u(0)=\sum_{k=1}^{m} c_{k} u\left(\tau_{k}\right), \tau_{k} \in(0, b) .
$$

where $0<\alpha<1,{ }^{c} D_{0^{+}}^{\alpha}$ denotes the Caputo fractional derivative of order $\alpha$, and $f: \mathcal{X} \rightarrow \mathcal{X}, K:\{(t, \sigma): 0 \leq \sigma \leq t \leq b\} \rightarrow \mathbb{R}^{+}, a, h:[0, b] \rightarrow \mathcal{X}$ are appropriate functions satisfying some conditions which will be stated later, $\tau_{k}, k=1, \ldots, m$ are prefixed points satisfying $0<\tau_{1} \leq \ldots \leq \tau_{m}<b$ and $c_{k}$ is real numbers.

The organization of this paper is as follows. In Section 2, we state some known notations and definitions and we also list the hypotheses which are used throughout this paper. Section 3 provides the proof of the existence of solution to the problem (1)-(2) in Banach space. Finally, an illustrative example is presented in Section 4.

\section{Preliminaries}

In this section, we present some essential notations, definitions and lemmas concerning fractional calculus and fixed point theorem. Let $J=[0, b]$ and $(\mathcal{X},\|\cdot\|)$ is a Banach space and $C(J, \mathcal{X})$ denotes the Banach space of all continuous functions from $J \rightarrow \mathcal{X}$ endowed with a topology of uniform convergence with the norm denoted by $\|$.$\| . Moreover, C^{n}(J, \mathcal{X})$ we denote the Banach space of all continuously differentiable functions up to order $(n-1)$ on $J$. 
Definition 1. ([13]) Let $\alpha>0$ and $g: J \rightarrow \mathcal{X}$. The left sided RiemannLiouville fractional integral of order $\alpha$ of a function $g$ is defined as

$$
I_{0^{+}}^{\alpha} g(t)=\frac{1}{\Gamma(\alpha)} \int_{0}^{t}(t-s)^{\alpha-1} g(s) d s, \quad t \in J,
$$

where $\Gamma($.$) is the Euler gamma function and I_{0^{+}}^{\alpha} g$ is exists for all $\alpha>0$. Moreover, $I_{0^{+}}^{0} g(t)=g(t)$.

Definition 2. ([13]) Let $n-1<\alpha<n, n \in \mathbb{N}$ and $g \in C^{n}(J, \mathcal{X})$. The left sided Caputo fractional derivative of order $\alpha$ of a function $g$ is defined as

$$
{ }^{c} D_{0^{+}}^{\alpha} g(t)=I_{0^{+}}^{n-\alpha} \frac{d^{n}}{d t^{n}} g(t), \quad t \in J,
$$

where $n=[\alpha]+1$, and $[\alpha]$ denotes the integer part of the real number $\alpha$.

Lemma 3. ([13, 16]) Let $\alpha, \beta>0$ and $g, p$ bee appropriate functions, then for $t \in J$, we have:

1. $I_{0^{+}}^{\alpha} I_{0^{+}}^{\beta} g(t)=I_{0^{+}}^{\alpha+\beta} g(t)=I_{0^{+}}^{\beta} I_{0^{+}}^{\alpha} g(t)$.

2. $I_{0^{+}}^{\alpha}(g(t)+p(t))=I_{0^{+}}^{\alpha} g(t)+I_{0^{+}}^{\alpha} p(t)$.

3. ${ }^{c} D_{0^{+}}^{\alpha}(g(t)+p(t))={ }^{c} D_{0^{+}}^{\alpha} g(t)+{ }^{c} D_{0^{+}}^{\alpha} p(t)$.

4. $I_{0^{+}}^{\alpha}{ }^{c} D_{0^{+}}^{\alpha} g(t)=g(t)-g(0), 0<\alpha<1$.

5. ${ }^{c} D_{0^{+}}^{\alpha} I_{0^{+}}^{\alpha} g(t)=g(t)$.

6. ${ }^{c} D_{0^{+}}^{\alpha} c=0$, where $c$ is a constant.

Lemma 4. ([1]) Let $\alpha>0$ and $g \in C(J, \mathcal{X})$. Then, $I_{0^{+}}^{\alpha} g \in C(J, \mathcal{X})$ and $I_{0^{+}}^{\alpha} g(0)=\lim _{t \rightarrow 0^{+}} I_{0^{+}}^{\alpha} g(t)=0$.

Lemma 5. ([17], Contraction mapping theorem) Let $(U, d)$ be a metric space. Then a mapping $T: U \rightarrow U$ is called a contraction mapping on $U$ if there exists a constant $k \in[0,1)$ such that $d(T(x), T(y)) \leq k d(x, y)$ for all $x, y$ $\in U$.

Lemma 6. ([17], Krasnoselskii fixed point theorem) Let $\mathcal{E}$ be bounded, closed and convex subset of a Banach space $\mathcal{X}$. Let $T_{1}, T_{2}: \mathcal{E} \rightarrow \mathcal{E}$ be operators such that $(i) T_{1} u+T_{2} v \in \mathcal{E}$, for every $u, v \in \mathcal{E} ;\left(\right.$ ii) $T_{1}$ is contraction; (iii) $T_{2}$ is compact and continuous. Then, there exists $z \in \mathcal{E}$ such that $z=T_{1} z+T_{2} z$. 


\section{Main results}

In this section, we shall demonstrate the existence result of the problem (1) - (2). For reader's comfort, a list of hypotheses is supplied as follows:

$\left(\mathbf{A}_{1}\right) a(t)$ and $h(t)$ are bounded and continuous on $J$.

$\left(\mathbf{A}_{2}\right) f: \mathcal{X} \rightarrow \mathcal{X}$ is continuous function and there exists $L>0$ such that

$$
\|f(u(t))-f(v(t))\| \leq L\|u-v\|, t \in J, u, v \in \mathcal{X} .
$$

$\left(\mathbf{A}_{3}\right) K: D \rightarrow \mathbb{R}^{+}$is continuous on $D$ with $K_{0}=\max \{|K(t, \sigma)|:(t, \sigma) \in D\}$, where $D=\{(t, \sigma): 0 \leq \sigma \leq t \leq b\}$.

First, we will state the following lemma.

Lemma 7. Let $0<\alpha<1, h, f$ and $K$ are continuous functions. If $u \in C(J, \mathcal{X})$, then $u$ satisfies the problem $(1)-(2)$ if and only if $u$ satisfies the integral equation

$$
u(t)=B \sum_{k=1}^{m} \frac{c_{k}}{\Gamma(\alpha)} \int_{0}^{\tau_{k}}\left(\tau_{k}-s\right)^{\alpha-1} H_{u}(s) d s+\frac{1}{\Gamma(\alpha)} \int_{0}^{t}(t-s)^{\alpha-1} H_{u}(s) d s,
$$

where $H_{u}(s):=\left[a(s) u(s)+h(s)+\int_{0}^{s} K(s, \sigma) f(u(\sigma)) d \sigma\right]$ and

$$
B=\frac{1}{1-\sum_{k=1}^{m} c_{k}}
$$

Proof. Let $u \in C(J, \mathcal{X})$ be a solution of $(1)-(2)$. By using Lemma 3, we get

$$
I_{0^{+}}^{\alpha}{ }^{c} D_{0^{+}}^{\alpha} u(t)=u(t)-u(0) .
$$

Applying the operator $I_{0^{+}}^{\alpha}$ on both sides of Eq. (1) and comparing result with Eq. (3) we obtain

$$
u(t)=u(0)+\frac{1}{\Gamma(\alpha)} \int_{0}^{t}(t-s)^{\alpha-1} H_{u}(s) d s .
$$

Now, we substitute $t=\tau_{k}$ in Eq. (4), and use nonlocal conditions Eq. (2) to get

$$
u(0)=\sum_{k=1}^{m} c_{k} u\left(\tau_{k}\right)=u(0) \sum_{k=1}^{m} c_{k}+\frac{1}{\Gamma(\alpha)} \sum_{k=1}^{m} c_{k} \int_{0}^{\tau_{k}}\left(\tau_{k}-s\right)^{\alpha-1} H_{u}(s) d s
$$




$$
=B \sum_{k=1}^{m} \frac{c_{k}}{\Gamma(\alpha)} \int_{0}^{\tau_{k}}\left(\tau_{k}-s\right)^{\alpha-1} H_{u}(s) d s
$$

Substituting Eq. (5) in Eq. (4), we get

$$
u(t)=B \sum_{k=1}^{m} \frac{c_{k}}{\Gamma(\alpha)} \int_{0}^{\tau_{k}}\left(\tau_{k}-s\right)^{\alpha-1} H_{u}(s) d s+\frac{1}{\Gamma(\alpha)} \int_{0}^{t}(t-s)^{\alpha-1} H_{u}(s) d s .
$$

On the other hand, by applying the fractional derivative operator ${ }^{c} D_{0^{+}}^{\alpha}$ on both sides of Eq.(6) and using Lemma 3, we have

$$
\begin{aligned}
{ }^{c} D_{0^{+}}^{\alpha} u(t) & ={ }^{c} D_{0^{+}}^{\alpha} I_{0^{+}}^{\alpha} H_{u}(t) \\
& =a(t) u(t)+h(t)+\int_{0}^{t} K(t, \sigma) f(u(\sigma)) d \sigma,
\end{aligned}
$$

which means that, Eq. (1) holds. Finally, we take $t \rightarrow 0$ in Eq. (6), and use Lemma 4, and can conclude that $u(0)=\sum_{k=1}^{m} c_{k} u\left(\tau_{k}\right)$. This completes the proof.

Next, we will prove the existence of solution for the problem (1)-(2) in the space $C(J, \mathcal{X})$ by means of Krasnoselskii's fixed point theorem.

Theorem 8. Assume that the hypotheses $\left(\mathbf{A}_{\mathbf{1}}\right),\left(\mathbf{A}_{\mathbf{2}}\right)$, and $\left(\mathbf{A}_{\mathbf{3}}\right)$ hold. If

$$
B \sum_{k=1}^{m} c_{k}\left[\rho+b K_{0} L\right] \frac{\left(\tau_{k}\right)^{\alpha}}{\Gamma(\alpha+1)}<1
$$

and

$$
\left[B \sum_{k=1}^{m} c_{k}\left(\tau_{k}\right)^{\alpha}+b^{\alpha}\right] \frac{\left(\rho+b K_{0} L\right)}{\Gamma(\alpha+1)}<\frac{1}{2},
$$

then the fractional integro-differential equation (1) - (2) has a solution in $C(J, \mathcal{X})$ on $J$.

Proof. Define the operator $\Upsilon: C(J, \mathcal{X}) \rightarrow C(J, \mathcal{X})$ by

$$
\Upsilon u(t)=B \sum_{k=1}^{m} c_{k} \frac{1}{\Gamma(\alpha)} \int_{0}^{\tau_{k}}\left(\tau_{k}-s\right)^{\alpha-1} H_{u}(s) d s+\frac{1}{\Gamma(\alpha)} \int_{0}^{t}(t-s)^{\alpha-1} H_{u}(s) d s .
$$

We need to analyze the operator $\Upsilon$ into sum two operators $P+Q$ as follows

$$
P u(t)=B \sum_{k=1}^{m} c_{k} \frac{1}{\Gamma(\alpha)} \int_{0}^{\tau_{k}}\left(\tau_{k}-s\right)^{\alpha-1} H_{u}(s) d s
$$


and

$$
Q u(t)=\frac{1}{\Gamma(\alpha)} \int_{0}^{t}(t-s)^{\alpha-1} H_{u}(s) d s .
$$

Now, we apply Lemma 6 in several steps:

$\operatorname{Step}(1):$ We prove that $P u+Q u^{*} \in S_{r} \subset C(J, \mathcal{X})$, for every $u, u^{*} \in S_{r}$.

Let $\mu_{0}=\|f(0)\|, \eta=\sup _{t \in J}\|h(t)\|, \rho=\sup _{t \in J}\|a(t)\|$ and choosing

$$
r \geq 2\left[B \sum_{k=1}^{m} c_{k}\left(\tau_{k}\right)^{\alpha}+b^{\alpha}\right] \frac{\left(\eta+b K_{0} \mu_{0}\right)}{\Gamma(\alpha+1)}
$$

such that $S_{r}=\{u \in C(J, \mathcal{X}):\|u\| \leq r\}$.

For the operator $P$ : By the previous assumptions, for $u \in S_{r}$ and $t \in J$, we have

$$
\|P u(t)\| \leq B \sum_{k=1}^{m} \frac{c_{k}}{\Gamma(\alpha)} \int_{0}^{\tau_{k}}\left(\tau_{k}-s\right)^{\alpha-1}\left\|H_{u}(s)\right\| d s .
$$

Note that

$$
\begin{aligned}
\left\|H_{u}(s)\right\| & \leq\|a\|\|u\|+\|h\|+\int_{0}^{s}\|K(s, \sigma)\|[\|f(u)-f(0)\|+\|f(0)\|] d \sigma \\
& \leq\left(\rho+b K_{0} L\right)\|u\|+\eta+b K_{0} \mu_{0} .
\end{aligned}
$$

Consequently,

$$
\begin{aligned}
\|P u(t)\| & \leq B \sum_{k=1}^{m} \frac{c_{k}}{\Gamma(\alpha)} \int_{0}^{\tau_{k}}\left(\tau_{k}-s\right)^{\alpha-1}\left[\left(\rho+b K_{0} L\right)\|u\|+\eta+b K_{0} \mu_{0}\right] d s \\
& \leq B \sum_{k=1}^{m} c_{k}\left[\frac{\left(\rho+b K_{0} L\right) r}{\Gamma(\alpha+1)}+\frac{\left(\eta+b K_{0} \mu_{0}\right)}{\Gamma(\alpha+1)}\right]\left(\tau_{k}\right)^{\alpha} .
\end{aligned}
$$

For the operator $Q$ : By the previous assumptions, for $u^{*} \in S_{r}$ and $t \in J$, we have

$$
\begin{aligned}
\left\|Q u^{*}(t)\right\| & \leq \frac{1}{\Gamma(\alpha)} \int_{0}^{t}(t-s)^{\alpha-1}\left[\left(\rho+b K_{0} L\right)\left\|u^{*}\right\|+\eta+b K_{0} \mu_{0}\right] d s \\
& \leq\left[\frac{\left(\rho+b K_{0} L\right) r}{\Gamma(\alpha+1)}+\frac{\left(\eta+b K_{0} \mu_{0}\right)}{\Gamma(\alpha+1)}\right] b^{\alpha} .
\end{aligned}
$$

From Eq. (12) and Eq. (13), we get

$$
\left\|P u+Q u^{*}\right\| \leq\|P u\|+\left\|Q u^{*}\right\|
$$




$$
\begin{aligned}
\leq & {\left[B \sum_{k=1}^{m} c_{k}\left(\tau_{k}\right)^{\alpha}+b^{\alpha}\right] \frac{\left(\rho+b K_{0} L\right) r}{\Gamma(\alpha+1)} } \\
& +\left[B \sum_{k=1}^{m} c_{k}\left(\tau_{k}\right)^{\alpha}+b^{\alpha}\right] \frac{\left(\eta+b K_{0} \mu_{0}\right)}{\Gamma(\alpha+1)} \\
\leq & \frac{r}{2}+\frac{r}{2}=r .
\end{aligned}
$$

This means that, $P u+Q u^{*} \in S_{r}$.

$\operatorname{Step(2):~We~prove~that~operator~} P$ is a contraction map on $S_{r}$.

Let us make $S_{r}$ as in step (1), by the preceding assumptions, then for $u, u^{*} \in S_{r}$ and for $t \in J$, we have

$$
\begin{aligned}
& \left\|P u(t)-P u^{*}(t)\right\| \\
\leq & B \sum_{k=1}^{m} c_{k} \frac{1}{\Gamma(\alpha)} \int_{0}^{\tau_{k}}\left(\tau_{k}-s\right)^{\alpha-1}\left\|H_{u}(s)-H_{u^{*}}(s)\right\| \\
\leq & B \sum_{k=1}^{m} c_{k} \frac{1}{\Gamma(\alpha)} \int_{0}^{\tau_{k}}\left(\tau_{k}-s\right)^{\alpha-1}\left[\rho\left\|u(s)-u^{*}(s)\right\|+b K_{0} L\left\|u(s)-u^{*}(s)\right\|\right] d s \\
\leq & B \sum_{k=1}^{m} c_{k}\left[\rho+b K_{0} L\right] \frac{\left(\tau_{k}\right)^{\alpha}}{\Gamma(\alpha+1)}\left\|u-u^{*}\right\| .
\end{aligned}
$$

By Eq. (8), we conclude that $P$ is contraction map on $S_{r}$.

$\operatorname{Step}(3)$ : We show that the operator $Q$ is completely continuous on $S_{r}$.

For this end, we consider $S_{r}$ defined as in step (1) and we prove that $\left(Q S_{r}\right)$ is uniformly bounded, $\left(\overline{Q S_{r}}\right)$ is equicontinuous and $Q: S_{r} \rightarrow S_{r}$ is continuous. Firstly, we show that $\left(Q S_{r}\right)$ is uniformly bounded. By step (1), then for any $u \in S_{r}$ and for each $t \in J$, we have

$$
\|Q u\| \leq\left[\frac{\left(\rho+b K_{0} L\right) r}{\Gamma(\alpha+1)}+\frac{\left(\eta+b K_{0} \mu_{0}\right)}{\Gamma(\alpha+1)}\right] b^{\alpha}:=\ell .
$$

This means that $Q S_{r} \subset S_{\ell}$, for any $u \in S_{r}$, i.e. the set $\left\{Q u: u \in S_{r}\right\}$ is uniformly bounded. Next, we will prove that $\left(\overline{Q S_{r}}\right)$ is equicontinuous. Let $u \in S_{r}$ and for $t_{1}, t_{2} \in J$ with $t_{1} \leq t_{2}$, we have

$$
\begin{aligned}
\left\|Q u\left(t_{2}\right)-Q u\left(t_{1}\right)\right\| \leq & \frac{1}{\Gamma(\alpha)} \int_{t_{1}}^{t_{2}}\left(t_{2}-s\right)^{\alpha-1}\left\|H_{u}(s)\right\| d s \\
& +\frac{1}{\Gamma(\alpha)} \int_{0}^{t_{1}}\left|\left(t_{2}-s\right)^{\alpha-1}-\left(t_{1}-s\right)^{\alpha-1}\right|\left\|H_{u}(s)\right\| d s
\end{aligned}
$$




$$
\begin{aligned}
\leq & \frac{\rho+b K_{0} L r}{\Gamma(\alpha)}\left[\int_{0}^{t_{2}-t_{1}}\left(\left(t_{2}-t_{1}\right)-s\right)^{\alpha-1} d s\right. \\
& \left.+\int_{0}^{t_{1}}\left(\left(t_{1}-s\right)^{\alpha-1}-\left(t_{2}-s\right)^{\alpha-1}\right) d s\right] \\
& +\frac{\left(\eta+b K_{0} \mu_{0}\right)}{\Gamma(\alpha)}\left[\int_{0}^{t_{2}-t_{1}}\left(\left(t_{2}-t_{1}\right)-s\right)^{\alpha-1} d s\right. \\
& \left.+\int_{0}^{t_{1}}\left(\left(t_{1}-s\right)^{\alpha-1}-\left(t_{2}-s\right)^{\alpha-1}\right) d s\right] \\
\leq & 2\left[\frac{\rho+b K_{0} L r+\eta+b K_{0} \mu_{0}}{\Gamma(\alpha+1)}\right]\left(t_{2}-t_{1}\right)^{\alpha},
\end{aligned}
$$

where $\eta, \mu_{0}$ and $\rho$ are defined as in step (1). Observe that, hfill $\left\|Q u\left(t_{2}\right)-Q u\left(t_{1}\right)\right\| \rightarrow 0$ when $\left|t_{2}-t_{1}\right| \rightarrow 0$, which means that $\left(\overline{Q S_{r}}\right)$ is equicontinuous. Finally, from the continuity of $h, f$ and $K$, we can directly reach that the operator $Q: S_{r} \rightarrow S_{r}$. So, $Q$ is relatively compact on $S_{r}$. Hence, by the Arzela-Ascoli theorem, the operator $Q$ is compact on $S_{r}$. An application of Lemma 6 shows that operator $\Upsilon=P+Q$ has a fixed point on $S_{r}$. So the fractional integro-differential equation $(1)-(2)$ has a solution $u(t) \in C(J, \mathcal{X})$. This proves the required.

\section{An example}

The present section contains an example to point up the key result established in Section 3. Consider the following nonlocal fractional integro-differential equation

$$
\begin{gathered}
{ }^{c} D_{0^{+}}^{\frac{1}{2}} u(t)=\frac{1}{6} \cos (t) u(t)+\frac{1}{3} \sin (t)+\int_{0}^{t} \frac{e^{-(\sigma-t)} u(s)}{4\left(3+e^{t}\right)} d s, \\
u(0)=\frac{1}{9} u\left(\frac{2}{3}\right) .
\end{gathered}
$$

Here, $c_{1}=\frac{1}{9}, \tau_{1}=\frac{2}{3}, \alpha=\frac{1}{2}, a(t)=\frac{1}{6} \cos (t), h(t)=\frac{1}{3} \sin (u), f(u(t))$ $=\frac{u(t)}{3+e^{t}}$, and $K(t, \sigma)=\frac{e^{-(\sigma-t)}}{4}$. For $u, v \in \mathcal{X}=\mathbb{R}^{+}$and $t \in[0,1]$. We can see that $\|f(u)-f(v)\| \leq \frac{1}{4}\|u-v\|$, and $K_{0}=\|K(t, \sigma)\|=\frac{e-1}{4}$.

So, the conditions $\left(\mathbf{A}_{\mathbf{1}}\right),\left(\mathbf{A}_{\mathbf{2}}\right)$ and $\left(\mathbf{A}_{\mathbf{3}}\right)$ are satisfied with $L=\frac{1}{4}, \mu_{0}=0$, $\eta=\frac{1}{3}$ and $\rho=\frac{1}{6}$. Further, it is easy to check that Eq. (9) and Eq. (8) hold too. In fact, $B=\frac{1}{1-c_{1}}=\frac{1}{1-\frac{1}{9}}=\frac{9}{8}$, a simple computation shows that

$$
c_{1} B\left[\rho+b K_{0} L\right] \frac{\left(\tau_{1}\right)^{\alpha}}{\Gamma(\alpha+1)} \approx 0.03<1
$$


and

$$
\left[c_{1} B\left(\tau_{1}\right)^{\alpha}+b^{\alpha}\right] \frac{\left(\rho+b K_{0} L\right)}{\Gamma(\alpha+1)} \approx 0.34<\frac{1}{2} .
$$

An application of Theorem 8 implies that problem (14)-(15) has a solution on $[0,1]$.

\section{References}

[1] M.S. Abdo, A.M. Saeed, H.A. Wahash and S.K. Panchal, On nonlocal problems for fractional integro-differential equation in Banach space, European J. of Scientific Research, 151 (2019), 320-334.

[2] M.S. Abdo and S.K. Panchal, Fractional integro-differential equations involving $\psi$-Hilfer fractional derivative, Adv. Appl. Math. Mech., 11 (2019), 1-22.

[3] M.S. Abdo and S.K. Panchal, Some new uniqueness results of solutions to nonlinear fractional integro-differential equations, Annals of Pure and Applied Mathematics, 16 (2018), 345-352.

[4] M.S. Abdo and S.K. Panchal, Weighted fractional neutral functional differential equations, J. Sib. Fed. Univ. Math. Phys., 11 (2018), 535-549.

[5] B. Ahmad and J.J. Nieto, Existence results for nonlinear boundary value problems of fractional integro-differential equations with integral boundary conditions, Boundary Value Problems, 1 (2009), 708576.

[6] B. Ahmad and S. Sivasundaram, Some existence results for fractional integro-differential equations with nonlinear conditions, Communications Appl. Anal., 12 (2008), 107-112.

[7] A. Anguraj, P. Karthikeyan and G.M. N'guérékata, Nonlocal Cauchy problem for some fractional abstract integro-differential equations in Banach spaces, Communications Math. Anal., 6 (2009), 31-35.

[8] K. Balachandran and F.P. Samuel, Existence of solutions for quasilinear delay integro-differential equations with nonlocal condition, Electronic J. Diff. Equ., 6 (2009), 1-7.

[9] K. Balachandran and J.J. Trujillo, The nonlocal Cauchy problem for nonlinear fractional integro-differential equations in Banach spaces, Nonlinear Anal. Theory Meth. Appl., 72 (2010), 4587-4593. 
[10] D. Delboso and L. Rodino, Existence and uniqueness for a nonlinear fractional differential equation, J. Math. Anal. Appl., 204 (1996), 609-625.

[11] K. Diethelm, The Analysis of Fractional Differential Equations, Lecture Notes in Mathematics \# 2004, Springer, Berlin (2010).

[12] D. Guo, V. Lakshmikantham, Nonlinear Problems in Abstract Cones, Academic Press (1988).

[13] A.A. Kilbas, H.M. Srivastava and J.J. Trujillo, Theory and Applications of Fractional Differential Equations, North-Holland Math. Stud., Elsevier, Amsterdam (2006).

[14] K.S. Miller and B. Ross, An Introduction to the Fractional Calculus and Differential Equations, John Wiley, New York (1993).

[15] S. Momani, Local and global existence theorems on fractional integrodifferential equations, J. Fract. Calc., 18 (2000), 81-86.

[16] S.G. Samko, A.A. Kilbas and O.I. Marichev, Fractional Integrals and Derivatives: Theory and Applications, Gordon and Breach, Yverdon (1993).

[17] Y. Zhou, Basic Theory of Fractional Differential Equations, World Scientific, Singapore (2014). 